Geograficando, vol. 15, n. ${ }^{\circ}$ 1, e051, mayo-octubre 2019. ISSN 2346-898X

Universidad Nacional de La Plata.

Facultad de Humanidades y Ciencias de la Educación. Departamento de

Geografía

\title{
Desarrollo territorial: hacia la construcción de una herramienta metodológica para abordar dinámicas de acumulación material e inmaterial
}

Territorial development: around a methodological construction to tackle the material and inmaterial accumulation dynamics.

Román Fornessi

Universidad Nacional de La Plata, Argentina

romanfornessi@gmail.com

\section{RESUMEN:}

Este artículo propone una herramienta metodológica para relevar dinámicas de desarrollo territorial. Para ello, retomamos primero una caracterización sobre el desarrollo vinculada a aspectos materiales, concepción de la que planteamos corrernos y complejizar la categoría desarrollo abonando a una perspectiva integral. Por eso arribamos a una definición de este concepto en tanto dinámicas materiales e inmateriales de acumulación. Para el abordaje de estas dinámicas planteamos cuatro dimensiones que recuperan el complejo entramado que conforma el territorio: político-institucional, socioproductiva, simbólico-identitaria y espacial.

Palabras clave: Desarrollo, Territorio, Dinámicas de acumulación material e inmaterial.

\begin{abstract}
:
The aim of this article is to provide a methodological tool to relieve territorial development dynamics. For this reason, we first go back to a development categorization relative to material aspects, conception from which we want to take some distance, and make the development category more complex helping to a comprehensive perspective; that is why we reach a definition of this concept involving material and non-material accumulation dynamics. To deal with these dynamics we suggest four dimensions to recover the complex grid that constitutes the territory.
\end{abstract}

KEYWORDS: Development, Territory, Acummulation dynamics.

\section{INTRODUCCIÓN. HACIA UNA CONCEPTUALIZACIÓN DEL DESARROLLO TERRITORIAL COMO DINÁMICAS DE ACUMULACIÓN MATERIAL E INMATERIAL}

Intentamos abordar el desarrollo desde una perspectiva integral, que no pone el énfasis en el crecimiento económico, aunque lo recupera como una de las dimensiones a considerar. Aquellas interpretaciones pueden ser halladas en las estrategias de intervención planificadas por alguna instancia estatal u organismo regional o internacional, tan difundidas en la segunda mitad del siglo pasado en América Latina (Vuotto, 2012). En el año 1950, durante la Conferencia de Montevideo, Raúl Prebisch por la CEPAL expone los principios para el desarrollo de la región en los que recomendaba a los países "determinar las metas específicas del desarrollo económico y establecer un orden de prioridades en su realización” (Furtado, 1985, p. 85, citado en Marinho, 1988, p. 13).

El contexto en el que se difundieron estas ideas se caracterizó por la pretensión de las economías latinoamericanas de encarar procesos de industrialización: el camino del desarrollo propuesto por la CEPAL se encontraba unido a la idea de "elevación de la productividad en toda la fuerza de trabajo" (Marinho, 1988, p. 20), dinámica que encontraba en ese objetivo industrializador su condición de existencia.

El pensamiento estructuralista de influencia keynesiana dominó la escena teórica hasta los años 70, cuando entra en crisis el paradigma del Estado como promotor de la industrialización. Este pensamiento proponía tres grandes objetivos: una política de equilibrio en la balanza de pagos, la promoción del desarrollo industrial como motor generador de empleo y la intervención estatal en materia de seguridad social (Ocampo, 2008).

A partir de la crisis del Estado de Bienestar, y en el marco de los debates teóricos y los análisis empíricos, hallamos que una línea crítica a este modelo provino de la escuela neoinstitucionalista, que 
propuso un enfoque basado en la desregulación del Estado, cuestionando entonces su centralidad en el nivel nacional como promotor del desarrollo y como coordinador social. Las ideas propuestas por esta corriente convergieron en el Consenso de Washington de fines de los 80, que impuso, entre otras políticas, control de gasto público, disciplina en la política fiscal para evitar grandes déficits, privatización de empresas públicas, reforma tributaria y traspaso de funciones de áreas como salud y educación a niveles subnacionales de gestión.

En los 90, lo importante para rescatar como clima de época es el cuestionamiento predominante al rol del Estado nacional como gestor y promotor de políticas de desarrollo. La descentralización propuesta por el documento mencionado estaba acompañada por una concepción que ponía en el centro de la escena la gestión desde lo local, escala que cobra relevancia y se torna objeto de planificación. Comienza a postularse que las diferencias no son estructurales, sino que dependen de la propia historia de los sistemas y su relación con el contexto. De este modo, adquirió un lugar sustancial el tema de la innovación como la posibilidad de generar la complejidad del sistema y desplegar su capacidad endógena, pero siempre en relación con un entorno con el cual interactuar en términos de intercambio de conocimiento, y que permite a su vez el establecimiento de redes. Para muchos teóricos, el lugar de realización de esas redes es el territorio (Chain, 2012).

La autora recupera dos fuentes teóricas centrales sobre el desarrollo local en América Latina: los trabajos de la CEPAL y las recomendaciones de políticas del BID y el Banco Mundial. En este período era común escuchar o leer sobre el paradigma del desarrollo local, o del crecimiento endógeno, vinculado a la planificación estatal basada en la participación de la comunidad en el diseño e implementación de políticas de desarrollo.

Para López (2015) los preceptos subyacentes en los modelos de planificación del desarrollo local que promueven los organismos internacionales de crédito para superar la crisis y/o generar desarrollo tienden a perpetuar el orden establecido y la lógica mundial de división del trabajo, promoviendo la hegemonía de los países pretendidamente desarrollados sobre los subdesarrollados. En este sentido también se expresa David Harvey (2003): “el cordón umbilical que une la acumulación por desposesión y la reproducción ampliada es el que está dado por el capital financiero y las instituciones de crédito, respaldado, como siempre, por los poderes del Estado" (2003, p. 152). Las estrategias de intervención propuestas por estos organismos crediticios fueron fórmulas unívocas para el desarrollo, cuyo sustrato conceptual y metodológico implica identificarlo directamente con la lucha contra la pobreza, lo que dio lugar a prácticas asistenciales focalizadas compatibles con el ajuste estructural y redujo los vínculos sociales a relaciones mercantiles (López, 2015; Coraggio, 2009).

En esta dirección, la CEPAL ha formulado estrategias de intervención para el desarrollo local en comunidades latinoamericanas con el fin de "promover el crecimiento económico de las sociedades locales de la región, a partir de la consolidación de factores de competitividad en el mercado local y regional” (ILPESCEPAL, 1999, p. 35).

Sin embargo, crecimiento económico no es homologable a desarrollo, porque la acumulación de capital no es la única dimensión a tener en cuenta -sobre la cual hay que trabajar y a la que hay que fortalecer-para generar dinámicas de desarrollo en una localidad (Casalis, 2008). Por eso, consideramos oportuno retomar la categoría de desarrollo territorial con el objeto de recuperar la complejidad de las relaciones, articulaciones, conflictos, y dinámicas de poder que se establecen en los territorios (García, 2010). Asimismo, creemos que es el concepto adecuado para dar cuenta de un proceso de acumulación integral en una comunidad a partir de las vinculaciones que establecen entre sí los actores. Con "acumulación integral” nos referimos a los intercambios políticos, económicos, sociales, culturales, institucionales, que son parte constitutiva de cualquier territorio, y que en este trabajo identificaremos como instancias de acumulación material e inmaterial, es decir, instancias de intercambio que incrementan cuantitativa y/o cualitativamente algún tipo de capital.

Nuestra perspectiva de territorio parte de una concepción que lo entiende como proceso complejo de interacción de dinámicas materiales y simbólicas de las que queremos dar cuenta a partir de la propuesta 
de trabajar con el concepto de desarrollo territorial. Cuando decimos "complejo" estamos poniendo de manifiesto el hecho de que existen actores que articulan entre sí, y esto debe ser relevado en nuestro trabajo.

Entendemos que es en esas vinculaciones e intercambios donde se gestan y se fortalecen las dinámicas de desarrollo de las que participan los actores territoriales, las cuales no se dan como resultado de un proceso natural, más bien necesitan ser construidas socialmente y esto implica poner en disputa el sentido conceptual y político de lo que se definirá como desarrollo en la práctica concreta y en el intercambio material e inmaterial entre los actores. Es allí donde centraremos nuestra mirada.

\section{¿QUÉ TIPO DE TERRITORIO ESTUDiamos?}

Este apartado tiene como finalidad ofrecer un punto de partida para pensar el territorio particular, al que observaremos en el marco de una conceptualización del territorio en general.

Interesa centrarnos en las corrientes que conciben al territorio como un producto de interrelaciones sociales que involucran intereses, conflicto, poder, dominación, disputa de sentidos, iniciativas contrahegemónicas, que lo entienden como producto de relaciones sociales históricas y como parte de un proceso dialéctico conformado por un entramado de relaciones que lo modifican, y que, a la vez, son por él modificadas (Laurelli y Finquelevich, 1990).

Además, estos conceptos ponen de manifiesto la constitución conflictiva y relacional del poder, que atraviesa al territorio y lo constituye: lógicas multiescalares, consecuencias de reformulaciones administrativas y jurisdiccionales y la conformación de actores contrahegemónicos son procesos que se desenvuelven al calor de dinámicas de poder.

Dichos supuestos los encontramos en desarrollos teóricos de la década de 1960. A modo de ejemplo recuperamos el trabajo de Giuseppe Dematteis (1967), quien reconoce que las interacciones territoriales son transescalares y se construyen en un proceso en el que intervienen diferentes lugares y personas. El autor indica que la territorialización es un proceso que trasciende las características del ambiente natural, supuesto iluminado por una concepción relacional de la geografía y del territorio que está fundado en procesos de comunicación, conflicto, intercambio y cooperación como formas de socialización.

Lo fundamental aquí es retomar la idea según la cual no hay territorio sin una trama de relaciones sociales. Esto significa relaciones y redes, articulaciones territoriales o tramas transescalares (Dematteis, 1985). El territorio se construye social y políticamente e indica una realidad material resultante de las relaciones sociales y las relaciones sociedad-naturaleza.

El territorio no hace referencia exclusivamente a un área geográfica o a una circunscripción políticoadministrativa, sino, más bien, a una articulación productiva, redes sociales y económicas, coaliciones sociales, instituciones y construcción de cierto sentido de pertenencia a una localidad determinada (Marsiglia, 2009).

En línea con ello encontramos enfoques recientes como el de Mabel Manzanal (2007) que nos indica que estudiamos territorios de la globalización, de la descentralización, y de la modernidad.

Estudiamos territorios de la globalización en tanto instancias donde se condensan procesos de vinculación global económica, social, política o cultural. Esto quiere decir que nuestros territorios están atravesados por lógicas globales, lo que no debe hacernos perder de vista los atravesamientos territoriales en el nivel local y regional.

En suma, que sean territorios de la globalización implica que son instancias de síntesis de dinámicas globales y espacios de intervención de agentes multiescalares.

Estudiamos, también, territorios de la descentralización. Esto por una cuestión histórica: durante las últimas dos décadas del siglo pasado, Latinoamérica asistió a procesos de descentralización de funciones estatales desde el nivel nacional a instancias provinciales o municipales. El argumento que imperó para llevar adelante el traspaso de funciones fue de tipo financiero. Es importante tener presente este contexto, sobre todo en el momento en que nuestro estudio se enfoque a relevar dinámicas de desarrollo territorial en 
localidades o municipios que se constituyeron como tales al calor de esas reformas, o que de alguna manera este traspaso de funciones desde el nivel nacional hacia el local haya marcado un punto de inflexión en la lógica administrativa y política de la localidad.

Estudiamos, a su vez, territorios de la modernidad. La modernidad implica que lo local se impregna de lo global: la presencia de lo universal en lo local (Touraine, 2005) es un aspecto a tener en cuenta para pensar el desarrollo territorial en una localidad. A partir de los procesos de globalización y descentralización se dan situaciones de contraposición de conflictos, sentidos en disputa, tensiones sociales que encuentran en la constitución de sujetos colectivos una modalidad de expresión y de lucha. En estos territorios operan actores con intereses y búsquedas que se contraponen o se complementan, generando territorialidades que reconstituyen al territorio en un proceso circular y dialéctico, de permanente transformación. Estas expresiones encuentran en distintos sujetos y actores formas de resistencia y de acción contrahegemónica (Touraine, 2005), con capacidad para gestar respuestas locales a procesos hegemónicos globales, en procura de construir otra realidad en los lugares donde viven. Santos (1996) afirma que los territorios posibilitan luchas que abren oportunidades centradas en la búsqueda de otras instituciones que implican otras formas de regulación de la realidad, otros esquemas, otras cosmovisiones.

Silveira (2011) plantea también una caracterización del territorio que permite entenderlo en su historicidad: lo piensa como "instancia donde se condensan acciones que han tenido lugar en el pasado o que son llevadas a cabo en el presente: cada acción le confiere actualidad al territorio (...) y por ello es una permanente reconstrucción de las cosas y las acciones" (2011, p. 3). El territorio no puede ser pensado exclusivamente en términos económicos, sino que debe ser comprendido como un híbrido entre materialidad y vida social. La autora plantea, entonces, al territorio como la instancia de síntesis donde conviven dinámicas temporales: acciones del pasado y del presente que se actualizan permanentemente en la formación territorial, "la acción contiene en su intencionalidad una idea de futuro y el territorio se vuelve un híbrido de pasado, presente y futuro, materialidad y acción" (2011, p. 6).

Sumado a la temporalidad, el territorio es expresión de espacialidad, nuevamente es Silveira quien ilumina este aspecto señalando las distintas escalas de manifestación: el lugar, el país, y el mundo. Ninguna de ellas puede pensarse aisladamente, ya que su existencia es relacional. La visibilidad de las fronteras entre cada instancia espacial es producto de la selección que realiza el investigador que estudia el territorio (Silveira, 2011). Milton Santos señala que es preciso definir la especificidad de cada formación espacial, no tanto como mecanismo de identificación por contraposición a las demás, sino más bien como forma de construir una coherencia metodológica que permita la recolección de datos del nivel espacial que al investigador le interesa (Santos, 1996).

Asimismo, es necesario poner de manifiesto que el territorio -también síntesis de temporalidades- tiene la posibilidad de condensar dinámicas en tiempos distintos, en un mismo proceso de territorialización. ¿Qué quiere decir esto? Que el territorio está en continua realización y re-realización, que los actores que operan en él y sobre él dejan huellas que perviven y los trascienden, y que contribuyen a la elaboración de nuevos procesos territoriales atravesados por temporalidades pasadas.

El territorio que estudiamos es, también, constituido identitariamente. Esta característica cobra especial relevancia ya que repone las temporalidades que condensa el territorio.

Arocena (1988) señala que en todo territorio existe una sociedad local, y utiliza este término para referirse al conjunto de actores que se involucran recíprocamente en vínculos materiales o simbólicos a lo largo del tiempo. Toda sociedad local construye una dimensión identitaria: "cada individuo se reconoce parte de un conjunto bien determinado, la expresión 'yo soy de...' expresa pertenencia a una comunidad determinada, que se caracteriza por conductas colectivas aceptadas, valores, normas, creencias generadas y transmitidas de generación en generación" (Arocena y Marsiglia, 2017, pp. 53-54). Hablamos de sociedad local, entonces, cuando el conjunto de actores territoriales comparte rasgos identitarios comunes, y muestra una manera de ser determinada que lo distingue. 
Este componente identitario encuentra su máxima expresión colectiva cuando se plasma en un proyecto común, no necesariamente en términos de planificación institucional, sino también evocando un horizonte compartido como comunidad. Lo que resulta interesante es entender al territorio como instancia misma de vínculos sociales en distintas escalas temporales y espaciales, como producto de dinámicas de conflicto y de poder territoriales.

Estas caracterizaciones nos abren el camino para pensar, entonces, el fenómeno del desarrollo territorial de una manera integral, en línea con esas definiciones sobre el territorio, y es por eso por lo que proponemos un abordaje multidimensional de dicho proceso, basado en dispositivos político-institucionales, socioproductivos, simbólico-identitarios y espaciales.

\section{APUNTES ANALÍTICOS SOBRE DESARROLLO TERRITORIAL}

Hasta ahora hemos hecho un recorrido que comenzó señalando lecturas que, entendemos, ejemplifican una concepción del desarrollo vinculada al crecimiento económico a partir de la planificación estatal o de algún organismo internacional. Luego abordamos algunas caracterizaciones del territorio -como categoría analítica- que nos permitieran organizar metodológicamente la investigación. La propuesta, ahora, es continuar este camino con una exploración sobre autores que han abordado el desarrollo territorial, cuyas lecturas se encuentran en línea con las cualidades del territorio señaladas anteriormente, y que nos servirán para identificar y delimitar las dimensiones con las que se abordará el trabajo empírico.

Para Magri y Rodriguez (2017) es importante entender el desarrollo territorial a partir de los procesos de transformación productiva y cambio institucional: el primero de ellos como resultado de la introducción de innovación en los procesos productivos, mientras que el cambio institucional resulta de la capacidad de los actores de conformar coaliciones para la construcción de proyectos colectivos perdurables en el tiempo. Estos elementos obligan a adoptar una perspectiva que considere la interacción entre los actores, tanto con relación a las actividades productivas como en el intercambio y construcción de capital simbólico. Las coaliciones hacen posible que se genere un ambiente de intercambio de saberes y de fortalecimiento de la socialización como resultado de un proceso de interacción.

La articulación entre los actores es también importante para Arocena y Sutz (2000), quienes denominan procesos de aprendizaje a instancias de reunión de personas para la resolución de problemas a partir de la interacción entre ellos, proceso en el cual se aplica, intercambia y crea conocimiento. Por su parte, Scott y Storper (2003) señalan que la promoción de la acción colectiva en los territorios por medio de la creación de espacios públicos donde diversos individuos se encuentran e intercambian conocimientos es fundamental para el desarrollo territorial.

Arocena y Marsiglia (2017) señalan que el concepto de desarrollo territorial se ha vuelto superador del de desarrollo local ya que da cuenta de la diversidad en la pluralidad: al utilizar el plural los territorios reconocen que cada territorio posee sus particularidades, pero que todos comparten características comunes que los hacen territorios modernos.

García (2010) sostiene que para analizar dinámicas de desarrollo territorial es menester identificar las relaciones conflictivas que constituyen al territorio y que deben ser observadas en los vínculos que los distintos actores territoriales construyen entre sí. Estos actores son los sujetos que de alguna manera intervienen en la localidad generando procesos de territorialización. Por territorialización se entiende a las acciones que modifican al territorio y lo reconfiguran permanentemente, y que, a la vez, son reconfiguradas por él en un ejercicio dialéctico.

Cravacuore (2006) propone el estudio de determinados actores que entiende como imprescindibles en un abordaje analítico del desarrollo territorial: unidades productivas como pueden ser los comercios, instituciones educativas, organismos estatales en el territorio y residentes de la localidad que puedan reponer la historia de la comunidad son actores cuyas construcciones de sentido pueden echar luz sobre las dinámicas 
de desarrollo a través de la complejidad de los vínculos entre ellos. Según este autor, la importancia reside en la posibilidad de que estas vinculaciones entre los actores abran paso a un proceso de sinergia territorial, entendida como la construcción colectiva de dinámicas de acumulación ampliada, es decir, no exclusivamente económica.

Raffestín (1993, citado en Haesbaert, 2013) elabora una conceptualización sobre las dos caras del territorio: como expresión material y como contenido simbólico. Esta distinción la propone para argumentar a favor de la idea según la cual el territorio es producido por el hombre, lo que evidencia su aspecto relacional. Esta lectura la complementamos con la de Montañez y Delgado (1998), quienes sostienen que es necesario analizar los procesos de construcción de territorialidad desde una perspectiva espacio-temporal, de manera tal que puedan recuperarse los efectos sobre el territorio de dinámicas de distintas temporalidades. Entonces, entenderemos por proceso de territorialización a acciones de los actores que de alguna manera intervienen en el territorio (según la definición que aportamos del mismo) alterándolo en su forma o en su contenido.

Identificamos a los actores territoriales como personas, instituciones, unidades productivas, el Estado mismo, que articulan de alguna manera entre sí, fundando constantemente procesos de territorialización que resultan en acumulación de algún tipo para la comunidad. Caracterizar estas dinámicas nos ayudará a entender al desarrollo territorial como proceso comprendido por instancias de acumulación social, política, institucional, cultural, económica y/o espacial.

Nos interesa complementar esta propuesta con la de Coraggio (2003) para pensar al desarrollo territorial como la puesta en marcha de un proceso dinámico de ampliación de las capacidades locales para mejorar sostenidamente la calidad de vida de la población, lo que incluye: componentes económicos (trabajo productivo, ingreso, satisfacción de necesidades, suficiencia y calidad de los bienes públicos), componentes sociales (integración en condiciones de creciente igualdad de oportunidades), componentes culturales (pertenencia e identidad histórica), componentes políticos (transparencia y legitimidad de las representaciones, mediaciones institucionales de los conflictos territoriales, decisiones colectivas).

Entonces, a la luz del desarrollo teórico y conceptual que propusimos al comienzo de este apartado, podemos afirmar que el objeto de estudio que debemos relevar con la herramienta metodológica que presentaremos aquí son las dinámicas de desarrollo territorial entendidas como instancias de acumulación política, institucional, cultural, social, económica, espacial, observables a partir de las articulaciones que existen entre los actores territoriales de la localidad, ya que entendemos que en esas vinculaciones se dan los procesos de acumulación descritos, entendidos como procesos de fortalecimiento de los actores locales en términos económicos, sociales o políticos.

\section{EL ABORDAJE METODOLÓGICO QUE PROPONEMOS}

El abordaje metodológico que proponemos surge como resultado de la articulación de dos conceptos operacionales. El primero es el de trama de valor, que nos aproxima al análisis de las interrelaciones entre los actores. El segundo, las dimensiones del desarrollo territorial, que nos permiten clasificar las dinámicas de acumulación material e inmaterial.

Tal como venimos argumentando, nuestro trabajo encuentra su fundamento en la noción de territorio como un proceso dinámico en el tiempo y en diferentes escalas, que recupera vinculaciones conflictivas, relaciones de poder y prácticas de apropiación. Creemos que esta concepción dinámica e integral del territorio, y concretamente del desarrollo territorial, puede ser pensada a partir del concepto de trama de valor elaborado por Caracciolo (2014) para considerar el valor agregado que generan emprendimientos de la economía social en términos no sólo materiales o económicos, sino también inmateriales, culturales, simbólicos, ambientales y políticos. La autora subraya que, si bien la propuesta es que estas tramas se desarrollen entre los actores locales, es fundamental también considerar las vinculaciones con actores de otras localidades de manera tal que, en un principio, se tome la ventaja que implica la proximidad física de los 
sujetos locales pero sin perder de vista que la relación con actores de otras localidades puede generar una vinculación a nivel regional en torno a un proyecto colectivo de construcción de poder.

De esta manera, la trama de valor está constituida horizontalmente por el conjunto de emprendimientos vinculados entre sí tanto verticalmente, por los intercambios entre las distintas instancias del proceso productivo, como en diagonal, por los servicios de apoyo técnico y financiero. Todos estos actores entramados construyen el territorio.

Resulta pertinente aclarar que la autora desarrolla el concepto de trama de valor pensando en las vinculaciones que se generan entre los distintos actores en el territorio. De ellos, al menos uno es un emprendimiento de la economía social. Más allá de la importancia que reviste para nosotros el concepto de trama de valor, el mismo está más vinculado al abordaje territorial que proponemos que al requerimiento de que haya un emprendimiento de la economía social en el territorio. Esto quiere decir que encontramos en la trama de valor la posibilidad de identificar procesos de acumulación política, social, cultural, institucional y económica.

En este sentido, nos servimos de este concepto en términos metodológicos, ya que entendemos nos permite echar luz sobre distintas dimensiones de las vinculaciones entre los actores, que responderían a lógicas de acumulación no exclusivamente económicas, por lo que, al observarlas, podremos describir dinámicas de desarrollo territorial en pequeñas localidades.

Dado que la trama de valor encierra articulaciones horizontales, verticales y en diagonal, se pone en evidencia la necesidad de pensar en distintos actores intervinientes en el territorio y las vinculaciones que existen entre ellos, y que permiten ponderar sus conocimientos y reconocer su interacción. Esto se encuentra en sintonía con la idea de Manzanal (2008), que rescata la importancia de las articulaciones y construcción de redes sociales entre los actores del territorio, y su potencial capacidad de gestación de un nuevo proceso de desarrollo.

En síntesis, con base en estas reflexiones arribamos a la idea de entender un proceso de desarrollo territorial integral multidimensional, que parte de un enfoque de territorio, entendido como un elemento cuyo rol es activo y estructurante de los procesos sociales (Massey, 2009). Se trata de una idea de territorio que no lo identifica con la plataforma topográfica donde suceden las cosas, sino con un proceso complejo, un entramado de relaciones, tensiones, conflictos entre actores que lo modifican y, a la vez, son por él modificados (Laurelli y Finquelevich, 1990).

\section{DIMENSIONES PARA EL ESTUDIO DEL DESARROLLO TERRITORIAL}

¿Cómo hacemos "observables" las dinámicas de desarrollo territorial en un territorio específico? Para responder a esta pregunta retomamos la clasificación analítica que realiza Casalis (2008), que nos servirá para la construcción metodológica de las dimensiones que abordaremos en el trabajo de campo (políticoinstitucional, socioproductiva, simbólico-identitaria). A estas dimensiones incorporamos la dimensión espacial para operativizar la definición de territorio adoptada.

Es importante resaltar que, si bien estas dimensiones se encuentran separadas analíticamente, en la realidad operan articuladamente: veremos cómo a partir de instancias de vinculación, dentro de lo que entenderemos como dimensión político institucional, se generan también instancias de acumulación socioproductiva o simbólico identitaria. Lo que proponemos de manera separada analíticamente aparece de forma interrelacionada empíricamente, sólo establecemos esta distinción a los fines de organizar y sistematizar el análisis de nuestro trabajo de campo y sus resultados. Además, esta distinción por dimensiones nos permite poner de manifiesto la complejidad de las dinámicas del desarrollo territorial, en línea con nuestra idea de territorio multidimensional y complejo. 


\section{DiMENSIÓN POLÍTICO-INSTITUCIONAL}

Refiere al conjunto de articulaciones que pertenecen al campo del conflicto de intereses entre los actores, las distintas mediaciones institucionales que existen en la localidad o fuera de ella pero que tienen injerencia en el territorio y la planificación de políticas públicas destinadas al mismo.

Autores como Boscherini y Poma (2006) reconocen la posibilidad de generar desarrollo a partir del conjunto de proyectos que una comunidad comparte y que son de intervención colectiva tanto en su formulación como en su implementación. Este tipo de iniciativa de carácter comunitario es analizada como una instancia de vinculación entre distintos actores territoriales a partir de las cuales se gestan articulaciones a través de las cuales se producen procesos de acumulación en algún sentido.

Esta dimensión puede ser relavada a través, por ejemplo, del análisis de instancias estatales que funcionan en el territorio como mediadoras de intereses o conflictos (pensamos, por ejemplo, en una delegación municipal). Cobra especial significado aquí el carácter conflictivo de estas vinculaciones, en tanto son espacios de disputa de intereses, y también de sentidos. Por ejemplo, las expectativas sobre lo que se puede esperar del Estado construyen una representación de lo que es o debería ser el Estado. A partir de esas acciones se va sedimentando un camino por el que transitan los actores, lo que elabora un entramado que perdura en el tiempo y a partir del cual se generan dinámicas de acumulación territorial, entendidas éstas como un proceso de fortalecimiento local que redunda en capital económico, social, político.

\section{DiMENSIÓN SOCIOPRODUCTIVA}

Remite a los actores económicamente productivos de la localidad. Aquí comprendemos a todos los sujetos que de alguna manera contribuyan con sus actividades a generar lazos productivos con otros actores de la comunidad. Con esto queremos decir que no solamente tomamos la definición de trabajo como actividad remunerada, sino que también pretendemos observar las vinculaciones que los sujetos construyen entre sí desde la producción en sentido amplio: en términos de actividad remunerativa o colaborativa.

Esta dimensión es abordable, por ejemplo, a partir de los comerciantes de la localidad, o por la interpelación a unidades productivas, o a sujetos que realizan actividades informales o participan de alguna actividad colaborativa en la comunidad.

Para que haya desarrollo es necesario que exista algún proceso de acumulación material que les permita a los individuos reproducirse. Además, a partir de esas dinámicas de acumulación material, por medio del trabajo entendido en un sentido amplio -como describimos más arriba-, también se generan instancias de acumulación inmaterial en las vinculaciones en que entran los actores proveyéndose de insumos, trabajando colaborativamente y vendiendo su propia producción.

Es innegable que esas instancias implican un mínimo de interacción entre los individuos, y será importante rescatar si estas experiencias de intercambio suceden hacia el interior de la localidad, hacia el exterior, o ambas.

\section{DIMENSIÓN SIMBÓLICO-IDENTITARIA}

Esta dimensión está relacionada con todos los dispositivos culturales que interpelan a los actores de la comunidad desde la historicidad de la localidad, su construcción identitaria y su pertenencia. En este sentido, apelamos a las construcciones que distintos actores elaboran sobre el "ser local". Así, es nuestra intención recuperar cómo, a partir de elementos territoriales presentes se apela a un pasado que sigue operando, y a un futuro que se construye en las representaciones de los propios individuos sobre el horizonte deseado como comunidad. 
¿Por qué es necesario abordar la dimensión simbólico-identitaria en un estudio sobre desarrollo territorial? Porque la intervención de varios actores en la historia de la localidad genera articulaciones entre ellos, operando en el sentido que describimos anteriormente sobre la capacidad de acumulación culturalidentitaria. Asimismo, la apropiación de los significantes y la elaboración de los significados entrará en conflicto según qué actor estemos mirando; ese encuentro conflictivo también es parte de una dinámica de desarrollo, pues en ese choque de significados se plantean y replantean instancias de realización social, de forma tal que no se presentan interpretaciones monolíticas, hecho que enriquece la dinámica de desarrollo territorial. Esta dimensión puede ser estudiada a partir, por ejemplo, del análisis de intervenciones culturales locales (murales, actividades performativas, monumentos, etc)

\section{DIMENSIÓN ESPACIAL}

Proponemos la incorporación de una cuarta dimensión que es la espacial, entendiendo que las dimensiones anteriormente mencionadas se articulan en un espacio determinado. Las representaciones espaciales de los actores también operan en las vinculaciones territoriales entre ellos. Entendemos representaciones espaciales en los términos de Ortega Valcárcel, quien retoma las perspectivas sobre espacio subjetivo y espacio vivido, y las define como las representaciones vinculadas a la experiencia práctica y mental, con el espacio como dimensión social: "La materialidad del espacio, desde estas perspectivas, es inseparable de las diversas interpretaciones que la sociedad construye para aprehenderla. El espacio no es una categoría ajena ni un objeto contrapuesto al sujeto social” (Ortega Valcárcel, 2000, pp. 345-346).

En este sentido, es necesario abordar la construcción espacial de la localidad que elaboran los referentes a consultar, pues allí también se vislumbran aspectos del orden del poder y de los conflictos de intereses.

Esto aporta una nueva caracterización sobre el tipo de territorio que estamos estudiando: en acuerdo con Silveira (2009) creemos que es pertinente identificar al territorio como espacio apropiado en el que se elaboran representaciones que influyen y atraviesan la vida de las personas que habitan esos lugares, y, por ende, de las vinculaciones que construyen entre ellos. El espacio es esencialmente social (Santos, 1986; Haesbaert, 2013); es una instancia que contiene a las demás y a la vez es contenida por ellas. Decimos que se resuelve en formas espaciales de expresión territorial, cuyo contenido es construido-deconstruidoreconstruido socialmente.

Milton Santos (1986) sostiene que a partir de las interacciones territoriales recuperamos la totalidad espacial, y descubrimos así la interdependencia entre los elementos: "cada acción no constituye un elemento independiente, sino un resultado del proceso social” (1986, p. 5). En este sentido, es interesante rescatar esas formas espaciales que condensan procesos sociales a partir de un entramado relacional de elementos territoriales. Es importante tener presente que estos elementos pueden ser leídos como variables, en el sentido metodológico del término: su significado no es inalterable y permanente, sino que adopta distintos valores, conforme la evolución de la historia y la relación con su contexto. Santos recupera esta característica bajo el nombre de mutabilidad del significado: "si bien cada elemento del espacio mantiene su nombre, su contenido y significación están siempre variando” (1986, p. 7).

\section{BALANCE Y CONSIDERACIONES FINALES}

En este artículo propusimos una herramienta metodológica para el relevamiento de dinámicas de desarrollo territorial, a partir de la comprensión de este fenómeno como instancias de acumulación material e inmaterial que se dan a partir de las articulaciones entre los actores territoriales de una comunidad. Esta construcción metodológica responde a una caracterización conceptual del desarrollo desde una perspectiva integral, es decir, no exclusivamente como crecimiento económico o acumulación material. Es por eso que recuperamos también la dimensión inmaterial de este proceso. A su vez, adherimos 
a una concepción del territorio que lo entiende en su complejidad, no en tanto continente o sustrato físico, sino como fenómeno social en sí mismo, por ende constituido (y constitutivo) de lógicas de poder, de dominación, de resistencia, de prácticas contrahegemónicas, y también identificado con los dispositivos físicos que conforman el paisaje. Esta complejidad con la que caracterizamos a ambos fenómenos, el desarrollo y el territorio, imprime con el mismo tinte al desarrollo territorial, y por eso consideramos que las cuatro dimensiones descriptas pueden allanar el camino para relevar el fenómeno en su multidimensionalidad.

Por último, huelga aclarar que esta herramienta metodológica la utilizamos en nuestro trabajo de investigación en el marco de la elaboración de la tesis de maestría, y consideramos que la puesta en práctica de ese dispositivo revelará sus potencialidades y sus limitaciones. Por ello, este artículo es también una invitación a hacer uso de esta herramienta para quienes estén interesados/as en relevar el fenómeno del desarrollo territorial, con el deseo no sólo de que sirva a los fines empíricos de una investigación que indagare estos temas, sino también de que a partir de los límites y posibilidades que exhiba en cada caso, la misma se pueda potenciar y perfeccionar.

\section{Bibliografía}

Arocena, José (1988) Discutiendo lo local: las coordenadas del debate. Cuadernos del CLAEH, n 45-46, pp. 7-16, Montevideo.

Arocena, José y Sutz, Judith (2000) Mirando los sistemas nacionales de innovación desde el sur. Presentación en la OEI.

Arocena, José y Marsiglia, Javier (2017) La escena territorial del desarrollo. Actores, relatos y politicas. Buenos Aires: Taurus.

Boscherini, Fabio y Poma, Lucio (2006) Más allá de los distritos industriales: el nuevo concepto de territorio en el marco de la economía global. En Boscherini, F y Poma, L (comps) Territorio, conocimiento y competitividad de las empresas: el rol de las instituciones en el espacio global. Buenos Aires: Niño y Dórila

Caracciolo, Mercedes (2014) Construcción de tramas de valor y mercados solidarios. En García, Ariel (comp) Espacio y poder en las Politicas de Desarrollo del siglo XXI. Buenos Aires: CONICET - GER.

Casalis, Alejandro (2008) El desarrollo territorial, un desafio para la construcción de un nuevo modelo de desarrollo (s/ d) [Texto digitalizado disponible para su consulta]

Chain, Leyla (2012) Vinculaciones entre conocimiento especializado y politicas de planificación para el desarrollo local: El caso de la Dirección de Asuntos Municipales de la UNLP en el proceso de asesoramiento del Plan Estratégico Brandsen (2002/2003) Tesis de Mestría. UNSAM - UAM.

Coraggio, José Luis (2003) El papel de la teoría en la promoción del desarrollo local. (Hacia el desarrollo de una economía centrada en el trabajo), en Coraggio, José Luis (2003) La Gente o el Capital. Desarrollo Local y Economia del Trabajo. Quito: Abya-Yala. Recuperado de https://www.coraggioeconomia.org/jlc/archivos\%20 para\%20descargar/11\%20El\%20Papel\%20de\%20la\%20teoria\%20en\%20DL.pdf

Coraggio, José Luis (2009) Territorio y economias alternativas. Trabajo presentado en I Seminario internacional Planificación Regional para el Desarrollo Nacional. Visiones, desafíos y propuestas. La Paz. Recuperado de https:/ /www.coraggioeconomia.org/jlc/archivos\%20para\%20descargar/Territorio_y_economias_alternativas.pdf

Cravacuore, Daniel (2006) La articulación de actores para el desarrollo local. En Rofman, Adriana y Villar, Alejandro. Desarrollo Local. Una revisión critica del debate. Buenos Aires: Universidad Nacional de Quilmes y Universidad Nacional de General Sarmiento - Espacio

Dematteis, Giuseppe (1967) L'organizzazione territoriale del Piemonte secondo l'I.R.E.S. Bollettino della Società Geografica Italiana, serie IX, Vol. VIII, ano C, Vol. CIV, 76-92. Disponible en http://societageografica.net/im ages/stories/1967.pdf

Dematteis, Giuseppe (1985). Le metafore della terra. La geografia umana tra mito e scienza. Milano: Feltrinelli 
Furtado, Celso (1985) Conferencia La fantasía organizada, Ed. Paz y tierra. Citado en Marinho, Luiz (1988) La CEPAL y las concepciones del desarrollo en América Latina. Documento de discusión interna CEPAL. Recuperado de https://repositorio.cepal.org/handle/11362/34704

García, Ariel (2010) Espacio y poder en las Políticas de Desarrollo del siglo XXI. García, Ariel (comp) Buenos Aires . Recuperado de https://dialnet.unirioja.es/descarga/articulo/6210969.pdf

Harvey, David (2003) El nuevo imperialismo. Reino Unido: Oxford University Press

ILPES (1999) Enfoques de desarrollo en América Latina, una revisión conceptual. Serie Desarrollo Económico (3). Naciones Unidas, CEPAL. Santiago de Chile

INTA (2007) Enfoque de desarrollo territorial: documento de trabajo no 1. - 1a ed. Buenos Aires: Instituto Nacional de Tecnología Agropecuaria - INTA. Programa Nacional de Apoyo al Desarrollo de los Territorios

Laurelli, Elsa y Finquelevich, Silvia (1990) Innovación tecnológica y reestructuración desigual del territorio: países desarrollados - América Latina. En Revista Interamericana de Planificación, (XXIII)84, 191-223.

López, Eduardo (2015) Desarrollo local: conceptos e instrumentos. Material de trabajo del seminario "Desarrollo local y participación" Facultad de Trabajo Social, UNLP. Septiembre de 2015.

Magri, Altäir y Rodriguez, Miranda (2017) La fábula de los tres hermanos y el desarrollo territorial en su complejidad multinivel. Consideraciones sobre el caso uruguayo en el contexto latinoamericano. En Cuadernos del CLAEH 2da. Serie, año 36, n 105, 99-126.

Manzanal, Mabel (2007) Territorio, poder e instituciones. Una perspectiva crítica sobre la producción del territorio. En Manzanal, Mabel; Arzeno, Mariana y Nussbaumer, Beatriz (comps.) Territorios en construcción: actores, tramas y gobierno entre la cooperación y el conflicto, Colección Cultura y Sociedad. Buenos Aires: CICCUS, 15-51.

Manzanal, Mabel (2008) Desarrollo territorial e integración nacional ¿Convergencia o divergencia? en Nun, Jose y Grimson, Alejandro (comp.) Territorios, identidades y federalismo, 183, Buenos Aires: Edhasa, 101-110

Marsiglia, Javier (2009) Cómo gestionar las diferencias: la articulación de actores para el desarrollo local. Tesis presentada de Maestría. UNSAM - UAM

Massey, Doreen (2009) Geometrías del poder y la conceptualización del espacio, CLACSO- Caracas, Centro Internacional Miranda. Citado en ILPES (2013)

Merino, Gabriel (2015) Luchas por la conducción del Estado en Argentina entre 1999 y 2003, el Grupo Productivo y el cambio de modelo. Misiones: Editorial Universitaria de la Universidad Nacional de Misiones.

Montañez, Gustavo y Delgado, Ovidio (1998) Espacio, territorio y región: conceptos básicos para un proyecto nacional. Cuadernos de Geografia (VII)1-2, 121-134

Ocampo, José (2008) Los paradigmas del desarrollo en la historia latinoamericana, en Machinea, José; Iglesias, Enrique y Altimir, Oscar (eds.), Hacia la renovación de los paradigmas del desarrollo en América Latina, Santiago de Chile: CEPAL y SEGIB

Ortega Valcárcel, José (2000) Los horizontes de la geografia. Barcelona: Ariel.

Potoko, Alejandra (2013) Entre el Estado y la sociedad: Procesos de transformación del territorio. El caso del barrio Sumay Pacha en la Quebrada de Humahuaca, REGISTROS, Mar del Plata, (9)10, 95-111. Recuperado de http s://periferiaactiva.files.wordpress.com/2015/08/entre-el-estado-y-la-sociedad-potocko.pdf

Raffestín, Claude (1993) Por una geografía del poder Ed Ática, Sao Paulo, Brasil. Citado en Haesbaert, Rogério (2013) Del mito de la desterritorialización la multiterritorialidad. En Revista Cultura y representaciones sociales, (8)15. México.

Santos, Milton (1986) Espacio y tiempo. En Cuadernos críticos de Geografia Humana, Universidad de Barcelona, $(X I I) 65$

Santos, Milton (1996) Metamorfosis del espacio habitado. Barcelona: Oikos-Tau

Scott, Allen y Storper, Michael (2003) Regions, globalization, development. En Regional Studies (37), 579-593.

Sili, Marcelo (2005) La Argentina rural. De la crisis de la modernización agraria a la construcción de un nuevo paradigma de desarrollo de los territorios rurales. Buenos Aires: Ediciones INTA 
Silveira, María Laura (2009) Espacio banal y diversidad: más allá de las demandas del príncipe. En Revista Huellas (13), $18-36$

Silveira, María Laura (2011) Territorio y ciudadanía: reflexiones en tiempos de globalización. En Uni-Pluri/versidad, (11)3. Recuperado de https://mail.google.com/mail/u/0/\#search/silveira/15da466394002elf?projector=1\& messagePartId $=0.1$

Touraine, Alan (2005) Un nuevo paradigma. Para comprender el mundo de hoy, Estado y Sociedad (135), Buenos Aires: Paidos

Vuotto, Mirta (2012) La Economía Social y las cooperativas en Argentina. En Voces en el Fénix, en línea http://www. vocesenelfenix.com/content/la-econom\%C3\%ADa-social-y-las-cooperativas-en-la-argentina 